\title{
"Off-target" effect of tamoxifen on the brain
}

Lee, C-M et al. PNAS 117, 19578-19589 (2020)

The tamoxifen (TAM)-inducible CreER/LoxP recombination system is widely used to generate time- and tissue-specific mouse mutants or to label specific cell populations for genetic fate mapping studies. However, this method is not without potential drawbacks, and several studies have reported adverse effects of TAM on tissues. A new study reveals that prenatal TAM exposure impairs cortical neurogenesis and patterning in mouse offspring. These findings suggest that TAM might have confounding effects in neural lineage tracing and genetic manipulation studies and call for caution when interpreting such data.

The highest single prenatal (embryonic day E7.5-E10.5) TAM dose that can be injected intraperitoneally in the pregnant dam without causing embryonic lethality is $\sim 1 \mathrm{mg}$. Here, the investigators administered $750 \mu \mathrm{g}$ of TAM per pregnant dam at E10 and studied the effects of TAM on embryonic brain development. Map2 immunostaining revealed that TAM dramatically thinned the cortical plate at E18, and data from a combination of single-cell RNA sequencing, quantitative in situ hybridization and immunostaining assays indicated that prenatal TAM impaired cortical neurogenesis by promoting precocious neuronal differentiation while blocking neural progenitor cell (NPC) proliferation. The team further demonstrated that TAM exerted these effects by dysregulating the Wnt-Dmrta2 signaling pathway, which has an important role in NPC fate maintenance and neural differentiation.

To study the potential influence of TAM on adult neurogenesis, the investigators employed an established TAM administration protocol for CreER/ LoxP-dependent gene targeting with injection of $2 \mathrm{mg}$ of TAM/day to 3-4-wk-old male mice continuously for 5 days.

Brain analysis 5 days after the last TAM administration showed that TAM greatly reduced neural stem cell proliferation in the subventricular zone and the dentate gyrus, the two areas where adult neurogenesis occurs.

"The TAM-inducible CreER/LoxP system laid the foundations of significant discoveries in adult and embryonic neural stem cell fate mapping, gene functions, as well as neuronal subtypes and activity-dependent neural circuitry. It seems that the side effect of TAM could be a vulnerable Achilles' heel for TAM-induced CreER/LoxP cell lineage tracing and/or genetic targeting studies," conclude the investigators in their report before adding that appropriate controls should be included in the experimental design to prevent data misinterpretation.

\section{Alexandra Le Bras}

Published online: 10 September 2020

https://doi.org/10.1038/s41684-020-0656-4

\section{NEW-Isolation Building/Study Facility Book your studies!}

Ridglan Farms Bred to be Better...Raised to be Best

For 50 years Ridglan Farms, Inc. has provided the highest quality beagles for biomedical research. The Ridglan colony has adhered to the strictest standards of quality in breeding, socialization, health care and colony management.

In toxicology, pharmacology and other fields of science, the RIDGLAN BEAGLE has earned its reputation of superiority.

- Superior Health

- Intense Socialization

- Outstanding Customer Service

- Housing

- Field Safety Studies
- Licensed Research Facility

- DOI Studies

- Beagle Serum, Plasma, Whole Blood

- Neuter/Spay upon request

- Littermate Selection 